\section{Intoxicações medicamentosas em crianças menores de cinco anos}

\section{Drug intoxication among children under five years old}

Guacira Corrêa de Matos 1

Suely Rozenfeld 2

Maria Elide Bortoletto 3

1 Mestrado. Escola Nacional de Saúde Pública. Fundação Oswaldo Cruz. Rua Leopoldo Bulhões, 1480. Rio de Janeiro, Rio de Janeiro, Brasil. CEP: 21.041-210 E mail: gmatos@ rio.matrix.com.br

2 Departamento de Epidemiologia. Escola Nacional de Saúde Pública da Fundação Oswaldo Cruz.

3 Sistema Nacional de Informações Tóxico-farmacológicas da Fundação Oswaldo Cruz.

\section{Resumo}

Objetivos: conhecer a magnitude das ocorrências de intoxicações em crianças menores de cinco anos, no Brasil.

Métodos: a partir de dados secundários do Sistema Nacional de Informações Tóxico-Farmacológicas (SINITOX) foram analisadas as freqüencias por classe terapêutica, seguimento e circunstâncias, dos casos de intoxicação em menores de cinco anos, nos anos de 1997 e 1998 em São Paulo e Rio Grande do Sul.

Resultados: no Brasil, foram registrados no período, 151.000 casos de intoxicações humanas. Quase um terço pôde ser associado a medicamentos, sendo que cerca de 40.000 casos ocorreram entre crianças menores de cinco anos. O maior número de casos correspondeu aos descongestionantes nasais, analgésicos, broncodilatadores, anticonvulsivantes, anti-histamínicos e contraceptivos orais. Os descongestionantes nasais, anticonvulsivantes, anti-histamínicos e expectorantes foram responsabilizados pelos óbitos. Conforme o SINITOX as principais circunstâncias dos eventos foram o acidente individual, o erro de administração $e$ o uso terapêutico.

Conclusões: as intoxicações medicamentosas representam um grave problema de saúde pública, que necessita intervenções no campo da prevenção e da promoção. Isto demonstra a necessidade de ações educativas, junto à população para reduzir o número de acidentes e injurias. As principais lacunas do SINITOX são o sub-registro, a articulação insuficiente com os serviços de saúde e a falta de padronização dos dados. Palavras-chave Toxicidade das drogas, Preparações farmacêuticas, Saúde Pública 


\section{Introdução}

Os medicamentos representam a principal causa de intoxicações humanas registradas no Brasil, segundo as estatísticas divulgadas, anualmente, pelo Sistema Nacional de Informações Tóxico-Farmacológicas (SINITOX).1-7

Até 1985, os registros apontavam os medicamentos como os principais responsáveis pelas intoxicações humanas. Em 1986, a notificação de envenenamentos por animais peçonhentos tornou-se compulsória em todo o território nacional. Elas assumiram até 1993 a primeira posição em número de registros. Entretanto, a partir de 1994, os medicamentos voltam à primeira posição, o que indica a magnitude do problema, especialmente se considerada a sub-notificação.

As estatísticas obtidas a partir dos registros dos Centros de Controle de Intoxicações do país, apresentam uma tendência de crescimento dos eventos causados por medicamentos que, em 1998, atingiram a proporção de $28,20 \%$ do total de notificações.1-7

Os grupos populacionais mais atingidos são as crianças entre zero a quatro anos e as mulheres. Entre as intoxicações medicamentosas, $35,17 \%$ dos casos notificados ocorreram em menores de cinco anos e, nesta faixa etária, os medicamentos foram responsáveis por $39,20 \%$ dos eventos tóxicos registrados.7

Outro aspecto importante é a maior frequiência relativa de intoxicações medicamentosas na região Sudeste, onde se consome mais medicamentos e onde estão 25.239 farmácias e drogarias, que correspondem, a 47,5\% do total oficialmente existente no país.

Igualmente, nos países industrializados do mundo ocidental, como Alemanha, França, Itália, Estados Unidos, Inglaterra e Canadá, os medicamentos respondem de um terço até a metade dos casos de intoxicação registrados, constituindo, portanto, um grave problema de saúde pública. A faixa etária mais atingida corresponde aos menores de cinco anos, predominantemente, entre os dois e três anos de idade. 8

Os registros da maioria dos países, entretanto, não refletem a real magnitude do problema, devido a fatores como a sub-notificação e a tendência de registro apenas dos casos mais agudos, com sinais clínicos mais exuberantes. 9

Apenas alguns países, como os Estados Unidos, com cerca de 66 centros de informação, distribuídos por todo o território nacional, conseguem alcançar um nível adequado de cobertura das intoxicações, o que permite traçar o perfil de morbidade e mortalidade das intoxicações medicamentosas e conhecer os fatores de risco associados. 10
No Brasil, a notificação de reações adversas a medicamentos ainda é incipiente, resumindo-se a algumas iniciativas isoladas em estados como o Ceará e São Paulo, inexistindo um sistema de informações de âmbito nacional. O registro de eventos tóxico-farmacológicos no país, embora ainda muito prejudicado pela sub-notificação, encontra-se em estágio mais avançado de organização, sob a coordenação nacional do SINITOX

$\mathrm{O}$ crescimento das intoxicações medicamentosas, especialmente entre os menores de cinco anos, vem sendo seguidamente destacado, pela equipe técnica do SINITOX, nas análises de dados encontradas nas publicações anuais das estatísticas de casos de intoxicações e envenenamentos no Brasil.1-7 Desta forma, a análise temporal dos dados secundários disponíveis e dos fatores associados aos eventos, pode vir a contribuir para o planejamento de programas e estratégias de intervenção, que visem à redução da morbidade e mortalidade por causas evitáveis.

O presente estudo objetiva caracterizar os fatores associados às ocorrências de intoxicações medicamentosas no Brasil, em crianças menores de cinco anos, e identificar as principais classes terapêuticas envolvidas, a evolução e as circunstâncias dos eventos.

\section{Métodos}

\section{População do estudo}

Consideraram-se os casos de intoxicações entre crianças de ambos os sexos, menores do que cinco anos de idade, entre 1997 e 1998, no Rio Grande do Sul e em São Paulo, Brasil.

\section{Fontes de dados}

Os dados foram obtidos, diretamente, junto ao Centro de Controle de Intoxicações de São Paulo (CCI, $\mathrm{SP})$ e ao Centro de Informações Toxicológicas do Rio Grande do Sul (CIT, RS), participantes do Sistema Nacional de Informações Tóxico-Farmacológicas. Foram empregadas, também, as estatísticas anuais publicadas pelo SINITOX referentes aos dados coletados pelo sistema nos anos de 1997 e 1998.6,7

As experiências pioneiras no controle e na produção de informações sobre intoxicações e envenenamentos foram os Centros de São Paulo e Rio Grande do Sul, que iniciaram suas atividades entre 1971 e 1980, anteriormente à criação do SINITOX. Devido à regularidade e ao volume de dados 
disponíveis, estes dois Centros foram selecionados para desenvolver o presente estudo.

O SINITOX foi constituído em 1980, pelo Ministério da Saúde, a partir da constatação, entre as prioridades do governo, da necessidade de criar um sistema abrangente de informação e documentação em Toxicologia e Farmacologia de alcance nacional. O SINITOX, em 1986, foi incorporado à estrutura do Centro de Informação Científica e Tecnológica da Fundação Oswaldo Cruz e tem, como principais atribuições, coordenar, compilar, analisar e disseminar dados e informações no campo das intoxicações e envenenamentos em todo o país. 11

Atualmente, existem trinta e dois Centros de Controle de Intoxicações em funcionamento, em 17 Estados, distribuídos pelo território nacional. Considerando a população de 1998, estimada em 161.790.311 habitantes, verifica-se que o Brasil possui 2,0 Centros por 10 milhões de habitantes. $\mathrm{Na}$ Região Norte este índice cai para 1,7 e na Região Nordeste 1,5; enquanto que na Região Sul o indicador cresce para 2,1, na Sudeste para 2,2 e na Centro-Oeste chega a 2,7 Centros por 10 milhões de habitantes. Desde 1985 as estatísticas dos Centros em atividade no país são divulgadas anualmente, pelo SINITOX e, em 1998, pela primeira vez, a participação atingiu $100 \%$, ou seja todos os Centros do país enviaram seus dados. ${ }^{7}$ Considera-se, entretanto, que a rede de Centros ainda é insuficiente, com uma excessiva população descrita para cada Centro, em todas as cinco regiões e com distribuição geográfica desequilibrada. 11

O SINITOX utiliza uma base de dados, ainda não completa e informatizada, desenvolvida em 1980, pelo CIT, RS, a partir do modelo americano da American Association of Poison Control Centers (AAPCC), com adaptações à realidade brasileira. Os formulários preenchidos pelos Centros de todo o país subdividem os agentes tóxicos em treze categorias: medicamentos, animais peçonhentos, animais não peçonhentos, produtos químicos industriais, pesticidas agropecuários, pesticidas domésticos, raticidas, domissanitários, produtos de toalete, plantas, alimentos, outros produtos e não determinado. A nova "Ficha de Notificação e Atendimento", em teste desde 1999, foi elaborada em 1997, por uma Comissão da Secretaria de Vigilância Sanitária, coordenada pelo SINITOX e composta por representantes da rede de Centros de Controle de Intoxicações. Ela contempla quatro novas categorias de agentes tóxicos: produtos veterinários, metais, drogas de abuso, outros animais venenosos e, também, classifica os animais peçonhentos em serpentes, aranhas e escorpiões. 12

\section{Variáveis selecionadas e classificação de} medicamentos

O SINITOX dispõe dos registros de intoxicações nacionais, regionais e dos Centros integrantes da Rede, classificados por agente, faixa etária, sexo, zona de ocorrência, circunstância e evolução.

A partir dos dados históricos disponíveis nos registros do SINITOX, observa-se que, na faixa etária analisada, as estatísticas apresentam distribuição aproximadamente uniforme entre os sexos. Observou-se também que os Centros analisados registram poucos eventos em zona rural, pois localizam-se em grandes áreas urbanas. Por estes motivos, as variáveis relativas a sexo e zona de ocorrência não foram consideradas para a realização do estudo. Entretanto, a bibliografia consultada assinala que a distribuição dos eventos apresenta variações importantes, quanto às diferentes idades contidas nesta faixa etária e por isso considerou-se essa variável importante.

A partir destas considerações, e da análise da "Ficha de Notificação e Atendimento", foram selecionadas como variáveis independentes, as classes de medicamentos, a idade, a evolução e a circunstância na qual ocorreu a intoxicação.

Para classificar os agentes responsáveis pelas intoxicações adotou-se o terceiro nível da Anatomical Therapeutic Chemical (ATC), por ser a classificação superníveis recomendada pelo Drug Utilization Research Group (DURG), da Organização Mundial da Saúde (OMS), para estudos de utilização de medicamentos. 13

Além da ATC, foram utilizados, como fontes de consulta, as listas das Denominações Comuns Brasileiras (DCB), das Denominações Comuns Internacionais (DCI) e da Relação Nacional de Medicamentos (RENAME), o Guia de Medicamentos, 14 o "Guia Terapêutico Guanabara", 15 o Dicionário de Especialidades Farmacêuticas (DEF)16 e fontes complementares do Centro de Apoio à Terapia Racional de Medicamentos (CEATRIM) da Universidade Federal Fluminense (UFF) em convênio com o Conselho Regional de Farmácia do Rio de Janeiro (CRF, RJ) que utiliza a base de dados Micromedex ${ }^{\circledR} .17$ Apesar desse esforço, restaram cerca de 30 medicamentos sem classificação, devido a dúvidas na grafia ou por não terem sido localizados nas fontes consultadas. 


\section{Análise dos dados}

Analisaram-se os casos de intoxicações, em menores de cinco anos, causados pelas dez primeiras classes de medicamentos, em freqüência absoluta e relativa, registrados em cada Centro de Controle selecionado, nos anos de 1997 e 1998. Incluíram-se também as informações constantes das publicações anuais do SINITOX. Ao analisar os dados disponíveis, segundo as classes de medicamentos, decidiu-se por trabalhar com o conjunto das dez principais categorias, cuja freqüência acumulada corresponde a cerca de 50 a $60 \%$ dos eventos registrados. Desta forma, as tabelas foram construídas utilizando-se estas classes. As demais classes estão assinaladas nas tabelas como "Outros". Decidiu-se também agregar os dados dos dois Centros, uma vez que a distribuição dos eventos analisados era similar.

\section{Resultados}

Os medicamentos constituíram o principal agente responsável por eventos tóxicos registrados pela Rede Nacional de Centros de Controle de Intoxicações, nos anos de 1997 e 1998. Em 151.100 intoxicações humanas registradas durante esse período, 43.729 $(28,94 \%)$ foram atribuídas aos medicamentos. O segundo agente, em quantidade de casos, corresponde ao grupo dos animais peçonhentos, com 34.249 registros $(22,67 \%) .6,7$

No mesmo período, as crianças com menos de cinco anos sofreram $15.599(35,67 \%)$ das intoxicações medicamentosas. Do total de 39.395 intoxicações humanas registradas nesta faixa etária, $39,60 \%$ foram causadas por medicamentos. 6,7

O registro de intoxicações é bastante desigual, quando comparadas as cinco regiões. As regiões Sul e Sudeste vêm contribuindo, ao longo do período de existência do SINITOX, com o maior volume de dados, não apenas devido à grande concentração populacional, mas também porque, nestas regiões localiza-se o maior número de Centros bem estruturados. Em 1997 e 1998, as Regiões Sul e Sudeste responderam juntas por $83,99 \%$ dos registros nacionais de intoxicações medicamentosas. 6,7

Os Centros de Controle que foram selecionados para o presente estudo estão localizados nas Regiões Sul e Sudeste, respectivamente, nos Estados do Rio
Grande do Sul e São Paulo. Conforme mencionado em métodos, a seleção foi feita com base no volume e regularidade dos dados, sendo estes Centros responsáveis, em 1997 a 1998, por 28,96\% dos registros de intoxicações humanas e por $35,42 \%$ dos registros de intoxicações medicamentosas de todo o Brasil.6,7

O Centro de Informações Toxicológicas do Rio Grande do Sul registrou, durante o período analisado, 6.335 casos de intoxicações medicamentosas, que correspondem a $27,41 \%$ das intoxicações humanas, assumindo a posição de principal agente dos eventos registrados pelo Centro. As crianças abaixo de cinco anos foram atingidas em 2.806 casos, ou $44,29 \%$ das intoxicações medicamentosas. Das 6.610 intoxicações nesta faixa etária, $42,45 \%$ foram causadas por medicamentos.

O Centro de Controle de Intoxicações de São Paulo está localizado na maior cidade brasileira e por isso, acolhe poucas ocorrências características de zona agrícola, como envenenamentos por pesticidas agropecuários e acidentes com animais peçonhentos. O Centro registrou, durante o período analisado, 9.161 casos de intoxicações medicamentosas, que correspondem a 44,33\% das intoxicações humanas. As crianças com menos de cinco anos foram vítimas em 4.062 casos, ou $44,34 \%$ das intoxicações por medicamentos. Das 8.907 intoxicações em crianças desta idade, $45,60 \%$ foram provocadas por tal agente.

Merece destaque o fato de que várias classes aparecem em ambos os Centros, nos dois anos analisados, como responsáveis por grande quantidade de casos, entre as quais, os descongestionantes nasais tópicos e sistêmicos (696 casos), os analgésicos (489 casos), os broncodilatadores (474 casos) e os anticonvulsivantes (397 casos). Quanto à primeira categoria, nos dados enviados pelo CIT, RS não foi possível distinguir os descongestionantes nasais sistêmicos dos tópicos. Isso não ocorreu para os dados do CCI, SP. Outra classe terapêutica importante para a análise é a dos contraceptivos orais (306 casos).

Ao decompor a totalidade da faixa etária estudada em intervalos de 12 meses de vida, observou-se uma grande concentração de casos nas idades de dois e três anos. Do total de 6.967 casos analisados, em menores de cinco anos, $4.147(58,23 \%)$ atingiram as crianças de dois e três anos de idade (Tabela 1). 
Casos registrados de intoxicações medicamentosas em menores de cinco anos por classes de medicamentos e idade. Centro de Controle de Intoxicações de São Paulo, e Centro de Informações Toxicológicas do Rio Grande do Sul, 1997 a 1998.

\begin{tabular}{|c|c|c|c|c|c|c|c|c|c|c|c|c|}
\hline \multirow{3}{*}{ Classe de medicamento } & \multicolumn{12}{|c|}{ Idade } \\
\hline & \multicolumn{2}{|c|}{$<1$ ano } & \multicolumn{2}{|c|}{1 ano } & \multicolumn{2}{|c|}{2 anos } & \multicolumn{2}{|c|}{3 anos } & \multicolumn{2}{|c|}{4 anos } & \multicolumn{2}{|c|}{ Total } \\
\hline & $\mathrm{n}$ & $\%$ & $\mathrm{n}$ & $\%$ & $\mathrm{n}$ & $\%$ & $\mathrm{n}$ & $\%$ & $\mathrm{n}$ & $\%$ & $\mathrm{n}$ & $\%$ \\
\hline Descongestionante nasal & 103 & 13,04 & 127 & 11,10 & 241 & 10,57 & 156 & 8,36 & 69 & 7,79 & 696 & 9,99 \\
\hline Analgésico & 50 & 6,33 & 47 & 4,11 & 148 & 6,49 & 164 & 8,78 & 80 & 9,03 & 489 & 7,02 \\
\hline Broncodilatador & 62 & 7,85 & 61 & 5,33 & 158 & 6,93 & 128 & 6,86 & 65 & 7,34 & 474 & 6,80 \\
\hline Anticonvulsivante & 51 & 6,46 & 48 & 4,20 & 137 & 6,01 & 108 & 5,78 & 53 & 5,98 & 397 & 5,70 \\
\hline Contraceptivo oral & 2 & 0,25 & 36 & 3,15 & 114 & 5,00 & 107 & 5,73 & 47 & 5,30 & 306 & 4,39 \\
\hline Outros & 522 & 66,07 & 825 & 72,11 & 1.482 & 65,00 & 1.204 & 64,49 & 572 & 64,56 & 4.605 & 66,10 \\
\hline Total & 790 & 100,0 & 1.144 & 100,0 & 2.280 & 100,0 & 1.867 & 100,0 & 886 & 100,0 & 6.967 & 100,0 \\
\hline
\end{tabular}

Fontes: Centro de Controle de Intoxicações de São Paulo, e Centro de Informações Toxicológicas do Rio Grande do Sul.

Quanto à evolução, os eventos tóxicos registrados pelos Centros são classificados, de acordo com a "Ficha de Notificação e Atendimento", em "Cura", "Cura não confirmada", "Seqüela", "Óbito", "Óbito outra causa", "Outra", "Ignorada".18 Ambos os Centros registram baixos índices de seqüelas e de letali- dade atribuídas aos medicamentos, na faixa etária estudada. Dos casos registrados pelo CIT, RS, $94,76 \%$ evoluíram para a cura, embora $53,46 \%$ estejam relacionados como cura não confirmada, o que pressupõe descontinuidade no acompanhamento dos casos (Tabela 2).

Tabela 2

Casos registrados de intoxicações medicamentosas em menores de cinco anos por idade e evolução no Centro de Informações Toxlcológicas do Rio Grande do Sul, de 1997 a 1998.

\begin{tabular}{|c|c|c|c|c|c|c|}
\hline \multirow[b]{2}{*}{ Idade } & \multicolumn{6}{|c|}{ Evolução } \\
\hline & $\begin{array}{l}\text { Cura } \\
\text { (n) }\end{array}$ & $\begin{array}{c}\text { Cura não confirmada } \\
\text { (n) }\end{array}$ & $\begin{array}{l}\text { Sequela } \\
\text { (n) }\end{array}$ & $\begin{array}{l}\text { Óbito } \\
\text { (n) }\end{array}$ & $\begin{array}{l}\text { Ignorada } \\
\text { (n) }\end{array}$ & $\begin{array}{c}\text { Total } \\
(n)\end{array}$ \\
\hline$<1$ ano & 80 & 128 & 1 & 1 & 19 & 229 \\
\hline 1 ano & 158 & 213 & 1 & 1 & 16 & 389 \\
\hline 2 anos & 414 & 521 & 3 & 1 & 40 & 979 \\
\hline 3 anos & 367 & 426 & 2 & - & 36 & 831 \\
\hline 4 anos & 140 & 212 & 3 & - & 23 & 378 \\
\hline Total & 1.159 & 1.500 & 10 & 3 & 134 & 2.806 \\
\hline$\%$ & 41,30 & 53,46 & 0,36 & 0,11 & 4,77 & 100 \\
\hline
\end{tabular}

Fonte: Centro de Informações Toxicológicas do Rio Grande do Sul. 
O CCI, SP incluiu nas tabelas enviadas uma classificação adicional, "Sem resposta", onde estão relacionados 68,56\% dos casos registrados em 1997 e 1998, prejudicando a análise dos dados e, também, pressupondo descontinuidade no acompanhamento.

Medicamentos não relacionados entre os dez principais causadores de intoxicações em crianças com menos de cinco anos também foram responsáveis por óbitos. O CIT, RS registrou um óbito em criança de um ano de idade atribuído a anti-histamínico, em 1997 e o CCI, SP registrou um óbito atribuído a expectorante, em 1998.
A principal circunstância envolvida é o acidente individual, que corresponde a $76,78 \%$ dos casos, seguida do erro de administração e do uso terapêutico. Essas três circunstâncias, juntas, são responsáveis por $92,02 \%$ dos casos. Chama atenção a freqüência relativa do erro de administração nos menores de um ano de idade, que corresponde a $39,11 \%$ dos casos registrados nesta idade. Os acidentes coletivos com medicamentos estão concentrados nas idades de dois, três e quatro anos (Tabela 3 ).

Tabela 3

Casos registrados de intoxicações medicamentosas em menores de cinco anos por idade e circunstância no Centro de Controle de Intoxicações de São Paulo e Centro de Informações Toxicológicas do Rio Grande do Sul, de 1997 a 1998.

\begin{tabular}{|c|c|c|c|c|c|c|c|c|c|c|c|c|}
\hline \multirow{3}{*}{ Circuntância } & \multicolumn{12}{|c|}{ Idade } \\
\hline & \multicolumn{2}{|c|}{$<1$ ano } & \multicolumn{2}{|c|}{1 ano } & \multicolumn{2}{|c|}{2 anos } & \multicolumn{2}{|c|}{3 anos } & \multicolumn{2}{|c|}{4 anos } & \multicolumn{2}{|c|}{ Total } \\
\hline & $\mathrm{n}$ & $\%$ & $\mathrm{n}$ & $\%$ & $\mathrm{n}$ & $\%$ & $\mathrm{n}$ & $\%$ & $\mathrm{n}$ & $\%$ & $\mathrm{n}$ & $\%$ \\
\hline Acidente individual & 169 & 21,39 & 851 & 74,38 & 2.002 & 87,81 & 1.607 & 86,07 & 721 & 81,38 & 5.350 & 76,78 \\
\hline Acidente coletivo & 2 & 0,25 & 1 & 0,09 & 15 & 0,66 & 23 & 1,23 & 12 & 1,35 & 53 & 0,75 \\
\hline Uso terapêutico & 136 & 17,22 & 86 & 7,52 & 62 & 2,72 & 55 & 2,95 & 43 & 4,86 & 382 & 5,49 \\
\hline Prescrição inadequada & 39 & 4,94 & 16 & 1,40 & 14 & 0,61 & 4 & 0,22 & 7 & 0,79 & 80 & 1,15 \\
\hline Erro de administração & 309 & 39,11 & 126 & 11,01 & 97 & 4,25 & 91 & 4,87 & 57 & 6,43 & 680 & 9,75 \\
\hline Automedicação & 47 & 5,95 & 13 & 1,14 & 14 & 0,61 & 13 & 0,70 & 8 & 0,90 & 95 & 1,37 \\
\hline Outra & 48 & 6,08 & 8 & 0,70 & 8 & 0,36 & 18 & 0,96 & 4 & 0,45 & 86 & 1,25 \\
\hline Ignorada & 40 & 5,06 & 43 & 3,76 & 68 & 2,98 & 56 & 3,00 & 34 & 3,84 & 241 & 3,46 \\
\hline Total & 790 & 100,0 & 1.144 & 100,0 & 2.280 & 100,0 & 1.867 & 100,0 & 886 & 100,0 & 6.967 & 100,0 \\
\hline
\end{tabular}

Fontes: Centro de Controle de Intoxicações de São Paulo, e Centro de Informações Toxicológicas do Rio Grande do Sul.

\section{Discussão}

O presente estudo analisou a magnitude das intoxicações medicamentosas em crianças menores de cinco anos, a partir das Estatísticas do Sistema Nacional de Informações Tóxico-Farmacológicas e dos registros do Centro de Informações Toxicológicas do Rio Grande do Sul e do Centro de Controle de Intoxicações de São Paulo, durante o período de 1997 a 1998.

Os medicamentos foram os principais responsáveis pelas intoxicações registradas no país. As crianças de zero a quatro anos foram as mais atingidas por intoxicações medicamentosas. Os medicamentos foram os principais responsáveis por intoxicações que atingiram a faixa etária dos menores de cinco anos. As crianças de dois e três anos foram as mais atingidas pelas intoxicações medicamentosas, dentro da faixa etária analisada.

As crianças entre dois e três anos de idade são as mais suscetíveis aos acidentes tóxicos, em todo o mundo. A curiosidade natural a essa faixa etária, associada ao desenvolvimento motor, aumentam o risco de exposição. Dos quatro anos em diante, as crianças já compreendem melhor as orientações dos adultos e são mais seletivas, quanto ao paladar. Nesta idade, os medicamentos envolvidos em eventos tóxicos são, geralmente, os de sabor agradável, adicionados de edulcorantes e com embalagens atraentes. 8 
Estudos realizados na Inglaterra indicam que as estatísticas de intoxicações em crianças caíram drasticamente, após a utilização de embalagens de segurança nos medicamentos e produtos químicos comercializados. 19

As cinco classes de medicamentos responsáveis pelo maior número de casos, em ambos os Centros, foram os descongestionantes nasais, os analgésicos, os broncodilatadores, os anticonvulsivantes e os contraceptivos orais.

Comparando os resultados obtidos com os registros de intoxicações no resto do mundo, foram encontradas referências a várias classes de medicamentos responsáveis por grande número de casos registrados no Brasil.10,19-25

Um estudo realizado na Inglaterra, acerca dos registros entre 1958 e 1977 apontou os antidepressivos, os analgésicos, os contraceptivos orais, os antinflamatórios e os suplementos dietéticos como os maiores responsáveis por intoxicações em crianças abaixo de dez anos. 19

Nos Estados Unidos, em 1997, os analgésicos, os antibióticos, as vitaminas, os preparados gastrointestinais e os hormônios foram os principais responsáveis pelas 305.749 intoxicações registradas em menores de seis anos. 10

O Centro de Informações Anti-Venenos de Portugal aponta os medicamentos como causadores de $30 \%$ das intoxicações em menores de cinco anos, sendo as principais classes envolvidas, os anti-histamínicos, os antibacterianos, os hormônios, os contraceptivos orais. 20

Na América Latina, o Centro Nacional de Control de Intoxicaciones de São José, localizado em Costa Rica, aponta os analgésicos, os benzodiazepínicos, os descongestionantes nasais, os antibióticos e os antiespasmódicos, como os principais causadores dos eventos. 22

No presente estudo, as causas ou circunstâncias das intoxicações medicamentosas, na faixa etária analisada, estão restritas a oito tipos, entre os 16 previstos na "Ficha de Notificação e Atendimento": "acidente individual", "acidente coletivo", "uso terapêutico", "prescrição inadequada", "erro de administração", "automedicação", "outra", "ignorada".18

$\mathrm{Na}$ Inglaterra, Wiserman et al. 19 apontaram como principais circunstâncias de intoxicações medicamentosas em crianças, os acidentes, a prescrição inadequada e a automedicação, sendo essa última, geralmente associada a produtos de venda livre.

Nos registros dos centros de controle brasileiros analisados neste estudo, o acidente individual é, também, a primeira causa em importância. Entretanto, como segunda causa encontra-se o erro de administração, que pode estar associado à pouca capacidade das mães compreenderem as instruções médicas e à inadequação das bulas dos medicamentos.

A inexistência de um sistema de informação e registro de reações adversas a medicamentos, provavelmente drena casos desta natureza para os Centros de Controle de Intoxicações. A própria "Ficha de Notificação e Atendimento" inclui a reação adversa, no item tipo de ocorrência. Esse fato pode explicar o uso terapêutico como terceira circunstância, em número de registros, ligada às intoxicações medicamentosas.

Os contraceptivos orais aparecem relacionados a sete acidentes coletivos, sendo a classe que apresenta maior quantidade de ocorrências desta natureza. Pode ser considerada a possibilidade de que as embalagens atraentes destes medicamentos, destinadas a estimular a mulher a não esquecer a ingestão diária do comprimido, sejam também estimulantes para brincadeiras. Outra observação importante refere-se ao aumento dos acidentes coletivos no ano de 1998, nos dois Centros e o fato de que haja registros unitários desses acidentes, levando a pensar em inadequação do registro ou atendimento de apenas uma das crianças vitimadas.

Os registros do CIT, RS, nos dois anos analisados, apontam os analgésicos, os descongestionantes nasais e os broncodilatadores como os principais responsáveis por acidentes individuais, respondendo juntos por $25,63 \%$, em 1997, e $22,97 \%$, em 1998 , dos casos onde está envolvida essa circunstância. As mesmas classes de medicamentos são responsáveis por $36,17 \%$, em 1997 , e $32,21 \%$, em 1998 , dos acidentes que tiveram, como causa determinante, o erro de administração.

O mesmo conjunto de classes de medicamentos nos registros do CCI, SP foi responsável por $22,18 \%$, em 1997 , e $21,76 \%$, em 1998 , dos acidentes individuais e por $33,33 \%$, em 1997 , e $30,14 \%$ dos erros de administração. Essas frequiências foram calculadas, considerando a soma dos analgésicos, broncodilatadores, descongestionantes nasais sistêmicos e tópicos, de modo a estabelecer comparação com o CIT, RS, onde essas duas últimas classes não estão individualizadas.

Analisando a terceira circunstância, em grau de importância, observa-se que, no CIT, RS, os descongestionantes nasais correspondem à classe envolvida no maior número de casos, correspondendo a $17,65 \%$, em 1997, e 10,45\%, em 1998, das intoxicações atribuídas ao uso terapêutico. Nos registros do CCI, SP, destacam-se os anticonvulsivantes que, em 1997, foram responsáveis por 20,49\% e, em 
1998 , por $14,08 \%$ dos casos determinados por essa circunstância.

Consideramos que a frequiência de casos esteja subestimada, dada a abrangência restrita do sistema, que não inclui casos muito leves ou muito graves. Eses últimos, se atendidos nas emergências dos hospitais, poderão escapar aos registros dos Centros, sobretudo quando os mesmos não prestam atividades assistenciais.

O sub-registro é outra importante lacuna apontada no Sistema, através de estudo realizado no período de 1987 a 1991, demonstrando que o número de notificações ao SINITOX representou menos da metade do total de pacientes internados por intoxicações e envenenamentos no país (133.729 casos notificados, 334.642 pacientes internados). Esse dado tem especial significado, considerando que as admissões hospitalares representam uma fração do total de casos reais de exposições tóxicas. ${ }^{11}$

Os Centros que apresentam maior volume de registros e regularidade de alimentação do Sistema, são o CCI, SP e o CIT, RS. Em 1998, esses dois Centros responderam por $28,5 \%$ do total de casos registrados no país. ${ }^{7} \mathrm{O}$ campo de pesquisa do CIT, RS, que trabalha apenas com informação, abrange a toxicologia clínica e epidemiológica. O CCI, SP dedica-se à pesquisa do abuso de drogas e, além da informação, exerce atividades de controle e tratamento, estando instalado no Hospital Municipal Dr. Artur Ribeiro de Saboya, no Jabaquara. ${ }^{11}$ Mesmo considerando tais características, pode haver certo grau de subestimação dos valores, dada a inexistência em nosso país de tradição de notificação, sobretudo de eventos não relacionados às doenças infecto-contagiosas.

A existência de grande número de casos com evolução assinalada como "cura não confirmada", "sem resposta" e "ignorada", pode indicar a descontinuidade de acompanhamento ou inadequações de registro.

Os medicamentos que levaram a óbito, em ambos os Centros, neste período, foram os descongestionantes nasais (dois óbitos), anticonvulsivantes (um óbito), anti-histamínicos (um óbito) e expectorantes (um óbito).

A principal causa ou circunstância dos eventos foi o acidente individual, seguida do erro de administração e do uso terapêutico.

O conjunto de dados apresentados demonstra que as intoxicações medicamentosas constituem um grave problema de saúde pública, que necessita de intervenções no campo da prevenção e da promoção, com vistas à redução do impacto provocado por estes agentes no quadro da morbi-mortalidade infantil.
O Centro de Informações Anti-Venenos do Instituto Nacional de Emergência Médica de Portugal26 preconiza as ações de prevenção em três níveis: 1) Prevenção primária-segurança e comercialização dos produtos, educação e legislação; 2) Prevenção secundária-prevenção das conseqüências, com tratamento precoce e eficaz (campo dos Centros de Informação e Controle] e 3) Prevenção terciária-tratamento das seqüelas, visando a redução da invalidez, recuperação e reintegração do paciente.

O padrão de consumo de medicamentos do nosso país é fortemente influenciado pela falta de controle em toda a cadeia do medicamento, desde a produção até a comercialização, levando ao consumo abusivo e irracional de produtos de venda livre e, mesmo dos que necessitam de receituário médico. Como conseqüência, verifica-se o crescimento de casos de intoxicações e envenenamentos, que sugere inadequações na produção, na circulação ou no uso de produtos farmacêuticos. 27

Os dados analisados no presente estudo apontaram os analgésicos e os descongestionantes nasais tópicos e sistêmicos, como os principais causadores de intoxicações em crianças de zero a quatro anos. Estes medicamentos são, em sua maioria, de venda livre e alvo da propaganda direta ao consumidor.

Um estudo realizado em três capitais brasileiras sobre o perfil da automedicação, apontou, também, os analgésicos e os descongestionantes nasais, como os medicamentos mais adquiridos, principalmente para uso familiar. ${ }^{28}$

É preciso que a Agência Nacional de Vigilância Sanitária reavalie a condição de venda sem prescrição médica dos produtos que contêm substâncias analgésicas, usadas, indiscriminadamente, para quadros leves e autolimitados. A compra condicionada à apresentação de receita, aliada a ações educativas, sinalizando para a sociedade sobre os cuidados com o uso desses produtos, poderia atuar favoravelmente na redução do número de acidentes com crianças.

No Brasil, o padrão de exigência legal, quanto às embalagens de produtos farmacêuticos, ainda é muito baixo. Os países desenvolvidos, desde a década de 70, adotaram a exigência da utilização de embalagens seguras, que reduziram a incidência de intoxicações acidentais em crianças. Desde 1994 existe, em tramitação no Congresso Nacional, projeto de lei que prevê a utilização de embalagem de proteção especial à criança (EEPC) em medicamentos e produtos químicos de uso doméstico.29 A aprovação de lei desta natureza, certamente, concorrerá para a redução dos eventos tóxicos causados por produtos 
farmacêuticos e químicos, que vitimam as crianças brasileiras em quantitativos crescentes, ano a ano.

O Sistema Nacional de Informações Tóxico-Farmacológicas, que coordena a Rede de Centros de Controle de Intoxicações distribuídos pelo território nacional, aponta uma série de lacunas que prejudicam a qualidade e a quantidade das informações, acerca das intoxicações e envenenamentos ocorridos no país. O sub-registro, a articulação insuficiente com os serviços de saúde e a falta de padronização dos dados estão entre as principais deficiências apontadas. 9

Efetivamente, durante a elaboração deste estudo, foi verificado que não há um sistema único de classificação de medicamentos, para orientar a coleta dos dados e o cruzamento das informações. Este problema atinge, inclusive os maiores Centros do país, apontando a necessidade de formação de grupo de trabalho, para desenvolver uma classificação, a ser adotada pelos Centros integrantes do Sistema.

A importância dos medicamentos, como agentes do quadro de morbidade infantil por causas evitáveis, demonstra a necessidade de ações educativas, tanto junto às crianças, quanto aos seus responsáveis, utilizando espaços públicos, creches, escolas,

\section{Agradecimentos}

Às equipes do Sistema Nacional de Informações Tóxico-Farmacológicas, ao Centro de Informações Toxicológicas da Fundação Oswaldo Cruz, ao Centro de Controle de Intoxicações de São Paulo e ao Centro de Informações Toxicológicas do Rio Grande do Sul.

\section{Referências}

1. SINITOX (Sistema Nacional de Informação Toxico-Farmacológicas). Estatística anual de casos de intoxicação e envenenamento. Brasil: 1992. Rio de Janeiro: Fundação Oswaldo Cruz (FIOCRUZ); 1993.

2. SINITOX (Sistema Nacional de Informação Toxico-Farmacológicas). Estatística anual de casos de intoxicação e serviços de saúde, comunidades e meios de comunicação. Esta proposta vai ao encontro de ações que já vêm sendo promovidas pelo próprio SINITOX, através de materiais educativos e divulgação de informações na imprensa. Igualmente, vários Conselhos Regionais de Farmácia e Sindicatos de Farmacêuticos, desde o início da década de 90 , promovem a Campanha pelo Uso Correto dos Medicamentos.

A Portaria 3.916 de 30 de outubro de 1998 do Ministério da Saúde, que aprova a Política Nacional de Medicamentos, inclui entre as diretrizes e prioridades, a Promoção do Uso Racional de Medicamentos, através de campanhas educativas, ações de farmacovigilância e incentivo aos estudos de farmacoepidemiologia. A implementação do conteúdo da Portaria é da responsabilidade dos três níveis de gestão do Sistema Único de Saúde e seu texto prevê a articulação intersetorial com entidades governamentais e não governamentais. 30

$\mathrm{O}$ presente estudo, relativo às intoxicações medicamentosas que atingem as crianças menores de cinco anos, visou apresentar alguns aspectos do problema, que possam vir a subsidiar estudos mais abrangentes e propostas para a redução de sua magnitude. envenenamento. Brasil: 1993. Rio de Janeiro: Fundação Oswaldo Cruz (FIOCRUZ); 1995.

3. SINITOX (Sistema Nacional de Informação Toxico-Farmacológicas). Estatística anual de casos de intoxicação e envenenamento. Brasil: 1994. Rio de Janeiro: Fundação Oswaldo Cruz (FIOCRUZ); 1996 
4. SINITOX (Sistema Nacional de Informação Toxico-Farmacológicas). Estatística anual de casos de intoxicação e envenenamento. Brasil: 1995. Rio de Janeiro: Fundação Oswaldo Cruz (FIOCRUZ); 1997.

5. SINITOX (Sistema Nacional de Informação Toxico-Farmacológicas). Estatística anual de casos de intoxicação e envenenamento. Brasil: 1996. Rio de Janeiro: Fundação Oswaldo Cruz (FIOCRUZ); 1998.

6. SINITOX (Sistema Nacional de Informação Toxico-Farmacológicas). Estatística anual de casos de intoxicação e envenenamento. Brasil: 1997. Rio de Janeiro: Fundação Oswaldo Cruz (FIOCRUZ); 1998.

7. SINITOX (Sistema Nacional de Informação Toxico-Farmacológicas). Estatística anual de casos de intoxicação e envenenamento. Brasil: 1998. Rio de Janeiro: Fundação Oswaldo Cruz (FIOCRUZ); FIOCRUZ;1999.

8. Scherz RG. Prevention of childhood poisoning- a community project. Pediatr Clin North Am 1970; 17: 713-7.

9. Possas CA, Bortoletto ME, Albuquerque DTC, Marques MB. Intoxicações e envenenamentos acidentais no Brasil: uma questão de saúde pública. Previdência Dados 1988; 3: 5-18.

10. TESS (Toxic Exposure Surveillance System). Annual Report of the American Association of Poison Control Centers: 1997. Washington, DC: TESS; 1998.

11. Marques MB, Bortoletto ME, Bezerra MCC, Santana RAL. Avaliação da Rede Brasileira de Centros de Controle de Intoxicações e Envenenamentos (CCIEs). Cad Saúde Pública 1995; 11: 560-78.

12. Bortoletto ME, Bochner R. Impacto dos medicamentos nas intoxicações humanas no Brasil. Cad Saúde Pública 1999; 15: 859- 69.

13. Capellà D, Laporte JR. Métodos empregados em estudos de utilização de medicamentos. In: Laporte JR, Tognoni G, Rozenfeld S, organizadores. Epidemiologia do medicamento. Rio de Janeiro: ABRASCO; 1989. p.95-113.

14. Zanini AC, Oga S. Guia de medicamentos. São Paulo: Ipex; 1998.

15. Korolkovas A. Dicionário terapêutico Rio de Janeiro: Guanabara Koogan; 1995

16. DEF (Dicionário de Especialidades Farmacêuticas). Rio de Janeiro: Publicações Científicas; 1999.

17. DRUGDEX®System [Healthcare series, v. 104 CD-Rom]. Englewood, Colorado: MICROMEDEX®; 1999.

18. SINITOX (Sistema Nacional de Informação Toxico-Farmacológicas). Manual de preenchimento da ficha de notificação e de atendimento: Centros de Assistência Toxicológica. Rio de Janeiro: Fundação Oswaldo Cruz (FIOCRUZ); 1997.
19. Wiserman HM, Guest K, Murray VSG, Volans G. Accidental poisoning in childhood: a multicentre survey. 1 . General epidemiology. Human Toxicol 1987; 6: 293-301.

20. Borges A. Portugal 1997. In: Annual Report. Lisboa: CIAV (Centro de Informações Anti-Venenos), Instituto Nacional de Emergência Médica; 1998.

21. CIAT (Centro de Información y Asesoramiento Toxicológico). Informe de actividades año 1996. Montevideo: Universidad de la Republica; 1997.

22. CNCI (Centro Nacional de Control de Intoxicaciones). Informe anual de consultas año 1998. San Jose: Hospital Nacional de Niños Dr. Carlos Sãenz Herrera; 1999.

23. Jaraczewsk W, Kotwica M. Acute poisoning with drugs: a review of data collected at the National Poison Information Centre during the period 1991-1995. Przegl Lek 1997; 54: 737-40

24. Kotwica M, Jarorz A, Kolacinsky Z, Rogaczwska A. Sources poisoning exposures in children during 19901995. An analysis of the National Poison Information Centre files. Int J Occup Med Environ Health 1997; 10 : 177-86.

25. Lambert H, Manel J, Bellow A, Kouch S. Morbidity and mortality from acute drug poisonig in France. Rev Prat 1997; 47: 716-20

26. Borges A. Prevenção em toxicologia. O Médico 1985; 112 908-16.

27. Rozenfeld S. Farmacovigilância: elementos para a discussão e perspectivas. Cad Saúde Pública 1998; 14: 237. 63.

28. Arrais PSD, Coelho HLL, Batista MCDS, Carvalho ML, Righi RE, Arnau JM. Perfil da automedicação no Brasil. Rev Saúde Pública 1997; 31: 71-7.

29. Brasil. Congresso. Câmara dos Deputados. Projeto de Lei $\mathrm{N}^{\circ} 4841$ 1999: determina a utilização de embalagem especial de proteção à criança - EEPC - em medicamentos e produtos químicos de uso doméstico que apresentem potencial de risco à saúde [autor] Fábio Feldman. [em tramitação].

30. Ministério da Saúde. Política nacional de medicamentos: anexo a Portaria no 3916 de 30 de outubro de 1998 Diário Oficial da União [DOU] 10 nov. 1998; Seção 1 , (215): 18-22

Recebido em 13 de novembro de 2001

Versão final reapresentada em 10 de julho de 2002

Aprovada em 20 de julho de 2002 